\title{
Rotation of an erupting filament observed by the STEREO EUVI and COR1 instruments *
}

\author{
A. Bemporad ${ }^{1}$, M. Mierla ${ }^{2,3,4}$, and D. Tripathi ${ }^{5, \star \star}$ \\ 1 INAF-Turin Astronomical Observatory, Pino Torinese (TO), Italy \\ e-mail: bemporad@oato.inaf.it \\ 2 Royal Observatory of Belgium, Brussels, Belgium \\ 3 Institute of Geodynamics of the Romanian Academy, Bucharest, Romania \\ ${ }^{4}$ Research Center for Atomic Physics and Astrophysics, Faculty of Physics, University of Bucharest, Romania \\ e-mail: marilena@oma.be \\ 5 Department of Applied Mathematics and Theoretical Physics, University of Cambridge, Wilberforce Road, \\ Cambridge CB3 0WA, UK \\ e-mail: durgesh@iucaa.ernet.in
}

Received 10 December 2010 / Accepted 5 May 2011

\section{ABSTRACT}

\begin{abstract}
On August 31, 2007, a prominence eruption was observed by the Solar TErrestrial RElations Observatory (STEREO) in the ExtremeUltraViolet Imager (EUVI) 304 images and later on, as the core of a three-part coronal mass ejection (CME) in images acquired by the inner STEREO coronagraph (COR1). Because they were covered by both STEREO spacecraft from right vantage points, these observations provide an excellent opportunity to perform a three-dimensional (3D) prominence reconstruction and study its evolution. We employed the tie-pointing technique to reconstruct the 3D shape and trajectory of the prominence, which has been followed from an heliocentric distance of $\sim 1.3$ up to $\sim 2.4 R_{\odot}$ during the first $1.3 \mathrm{~h}$ of eruption. Data show evidence for a progressive clockwise prominence rotation by $\sim 90^{\circ}$ occurring not only in the early phase of the eruption sampled by EUVI, but also at larger heliocentric distances as seen by COR1. Interestingly, a counter-clockwise rotation of the filament was observed in $\mathrm{H} \alpha$ images in the week before the eruption; the filament does not show a twisted shape. In the same period, the potential field extrapolated at different times shows a clockwise rotation of closed lines overlying the filament. This suggests that a magnetic helicity storage occurred not in the filament itself, but in the global magnetic field configuration of the surrounding corona. Moreover, close inspection to the high-resolution EUVI images revealed a small scale helical feature along the erupting prominence. The sense of rotation of this helix agrees with the observed prominence rotation, providing evidence for the conversion of twist into writhe. The observed rotation of an erupting prominence, if representative of the flux rope rotation, may have a strong impact on the definition of geo-effectiveness of CMEs for space weather forecasting purposes.
\end{abstract}

Key words. Sun: filaments, prominences - Sun: corona - Sun: coronal mass ejections (CMEs)

\section{Introduction}

Erupting prominences are one of the best proxies for coronal mass ejections (CMEs; Gopalswamy et al. 2003). Therefore, it is important to study the erupting prominences during the early phase of evolution, which may provide some clues regarding the geo-effectiveness of CMEs. It has been suggested that CMEs are geo-effective if they carry a southward component in their magnetic field. Based on the photospheric magnetic field distribution, guesses have been made regarding the geo-effectiveness of CMEs. However, these guesses have often been wrong. This is likely because CME structures show substantial rotation during their evolution in the corona (e.g., Yurchyshyn et al. 2009) and possibly in the heliosphere (e.g. Lynch et al. 2009a).

It is well known that erupting filaments generally exhibit helical structures (e.g., Athay et al. 1983; Rust \& Kumar 1994) and are sometimes observed to undergo a rotation about the vertical direction as they rise (e.g., Kurokawa et al. 1987). For this reason, an important quantity often used to characterize the degree

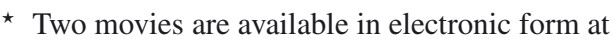
http://www . aanda.org

$\star \star$ Present address: Inter-University Centre for Astronomy and Astrophysics, Post Bag 4, Ganeshkhind, 411007 Pune, India.
}

of instability of filaments is the magnetic helicity $H$ (Berger \& Field 1984), defined as $H=\int_{V} \boldsymbol{B} \cdot \boldsymbol{A} \mathrm{d} V$, where $V$ is the volume where $H$ is measured, $\boldsymbol{B}$ is the magnetic field and $\boldsymbol{A}=\nabla \times \boldsymbol{B}$ is the vector potential. The magnetic helicity mainly quantifies to which degree a set of magnetic flux tubes are sheared and/or wound around each other, and it can be written as the sum of "twist" and "writhe": the twist quantifies how the fieldlines wind about the magnetic axis of the rope, whereas the writhe quantifies the helical deformation of the axis itself. The observed rotation of the erupting filament is in general interpreted as a conversion of twist into writhe in a kink-unstable magnetic flux rope (see e.g. Zhou et al. 2006). It has also been shown that an important condition for the formation and maintenance of a filament is a "handedness" property known as chirality $\chi$, which requires the filaments to be either of two types: dextral or sinistral (see review by Martin 1998, and references therein); $H$ is a quantitative, mathematical measure of $\chi$. Consistent with this interpretation, the rotation is usually found to be clockwise (as viewed from above) if the post-eruption arcade has right-handed helicity, but counterclockwise if it has left-handed helicity.

Helicity $H$ plays a very important role in the filament destabilization: for instance, the so-called "helical kink" instability of a magnetic flux rope is expected to occur when the twist exceeds 
a critical value (Hood \& Priest 1981). From a theoretical point of view in a high magnetic Reynold number plasma (i.e., in ideal MHD) $H$ is a conserved quantity (even taking into account the effects of magnetic reconnection; Berger \& Field 1984) and observations show that erupting filaments, different from stable filaments, exhibit large-scale twist or writhe, clearly appearing as helical-like patterns and rotations during their eruptions. Hence, it is generally believed that prominence eruptions and resulting CMEs are the most efficient way the Sun has to globally "carry out" the excess of helicity built in its interiors by the solar dynamo.

Indeed, the occurrence of transient coronal (soft X-ray or EUV) "S-shaped" structures (namely sigmoids), associated to higly twisted loop systems, is often correlated with a tendency of the active region to erupt into a coronal mass ejection (CME) (Canfield et al. 1999), and many attempts have been made to determine the relationship between coronal sigmoids and erupting flux ropes (see e.g. Green et al. 2007). For these reasons, the characterization of prominence rotation plays an important role in understanding how the eruptions are triggered. Unique information on the prominence and CME 3D motions have been derived in the last $\sim 12$ years from the analysis of spectroscopic observations acquired by the SOHO UV Coronagraph Spectrometer (UVCS; see Kohl et al. 1995). Spectroscopic observations of CME cores provided for the first time informations both on the plasma velocity components along the line of sight (LOS - from the line Doppler shifts) and in the radial direction (thanks to the Doppler dimming/pumping effect, see Noci et al. 1987). The first CME observed by UVCS provided evidence for rotation of the velocity vector associated with helical motions of plasma around an erupted magnetic flux tube (Antonucci et al. 1997). The line intensity morphologies and line profile Doppler shifts have been used to infer the handedness of untwisting lefthanded (Ciaravella et al. 2000) and right-handed (Suleiman et al. 2005) CME helixes and to verify that their handedness agreed with that of the pre-CME structures.

More recently, Green et al. (2007) have examined a number of filament eruptions associated with transient sigmoid features, which are characterized by their rotation during eruption. The authors find that sheared-arcade models as well as flux rope models agree with the observed relationship between positive (negative) helicity and clockwise (counterclockwise) rotation. Similar rotation is also seen in numerical simulations of erupting flux ropes (Fan \& Gibson 2004; Török et al. 2004). Interesting information on the occurrence of strong rotation of an erupting quiescent polar crown prominence, which agrees with the idea of untwisting of a helical flux rope, has recently been reported by Thompson (2011) for an event on June 6, 2007.

In this work we report on the observed rotation of a prominence during its eruption and, later on, in the resulting CME core. The 3D trajectory of the prominence reported here was studied with images acquired by the SECCHI EUV Imager (EUVI; see Wuelser et al. 2004) and the COR1 coronagraph (see Howard et al. 2008), both aboard the two twin Solar TErrestrial RElations Observatory (STEREO; see Kaiser et al. 2008) spacecraft. The paper is organized as follows: in the second and third sections we describe the event's general morphology (2) and the data analysis techniques we employed for this study (3). Next, our results are described in Sect. 4 and discussed in Sect. 5.

\section{General description of the event}

A prominence eruption was observed in EUVI 304 images on 31 August, 2007, starting from 19:00 UT (Fig. 1, top panels).
The paired EUVI telescopes onboard STEREO observe the fulldisk EUV chromosphere and corona with four bandpass channels which, similarly to those of SOHO/EIT, have peak responses around $304 \AA\left(\simeq 6-8 \times 10^{5} \mathrm{~K}\right.$, primarily the He II line $)$, $171 \AA\left(\simeq 10^{6} \mathrm{~K}\right.$, primarily Fe Ix/x), $195 \AA\left(\simeq 1.6 \times 10^{6} \mathrm{~K}\right.$, primarily Fe xII $)$, and $284 \AA\left(\simeq 2.5 \times 10^{6} \mathrm{~K}\right.$, primarily Fe xv). The field of view of $2048 \times 2048$ pixel images goes up to $1.7 R_{\odot}$, with a two-pixel spatial resolution by 3.2 arcsecs; these images are acquired with different cadences for different filters, going typically from 2.5 to $10 \mathrm{~min}$. On August 31, 2007, the separation angle between the STEREO spacecraft was $28.35^{\circ}$ (at 19:00 UT), so that the prominence was observed nearly at the limb from STEREO-B and partially on-disk from STEREO-A (Fig. 1). The EUVI 304 sequence shows in first approximation a simple loop-like feature expanding outward from the southwest limb, which was observed off-limb also from STEREO-A by 20:20 UT (Fig. 1, 20:23 UT panel) and began to exit from the STEREO-B field of view around 20:50 UT (Fig. 1, 20:53 UT panel).

The prominence was also seen later on in COR1 images as the core of a bright CME (Fig. 1, bottom panels). The COR1 telescopes observe the white-light K-corona from 1.4 to $4 R_{\odot}$ in a waveband $22.5 \mathrm{~nm}$ wide centered on the $\mathrm{H} \alpha$ line at $656 \mathrm{~nm}$ (Thompson \& Reginald 2008). The COR1 images are $1024 \times 1024$ pixel, with a pixel-limited spatial resolution of $3.75 \mathrm{arcsec} / \mathrm{pixel}$ and a temporal resolution of $10 \mathrm{~min}$. Owing to telemetry restrictions, the COR 1 images are typically $2 \times 2$ binned onboard before downloading to ground. Images acquired by COR1 on August 31, 2007, show a CME with the classical three-part structure, i.e., a semicircular bright front enclosing a dark cavity and an expanding prominence. Unfortunately, no $\mathrm{SOHO} / \mathrm{LASCO}-\mathrm{C} 2$ images are available for this event because of the coincidence with a SOHO keyhole, hence this CME is not included in the LASCO CME catalog. Because of the different fields of view covered by the COR1 telescopes onboard the twin STEREO spacecraft, the prominence first enters in the STEREO-A field of view (20:45 UT) and later on in the STEREO-B field of view (20:51 UT). In the subsequent images, the prominence material can be followed in the COR1 field of view until $\sim 22: 30 \mathrm{UT}$, while later on the prominence becomes too faint to be followed, because of plasma expansion. Two composite movies showing the evolution of the prominence in EUVI and COR1 field of views as seen from STEREO-A and -B are available online (see Figs. A.1 and A.2 in the appendix).

In the days before the eruption, a dark filament is visible in $\mathrm{H} \alpha$ images. In particular, full-disk $\mathrm{H} \alpha$ images available from the Mauna Loa Solar Observatory show an elongated $\mathrm{H} \alpha$ filament located in the southern hemisphere (see sequence in Fig. 2). The filament is U-shaped and it is possible to identify a westward and an eastward branch, called W- and E-branch, respectively. The EUVI images acquired by STEREO-A clearly show that the August 31, 2007, eruption involved only the E-branch, which is located close to the limb in the hemisphere visible from the Earth on August 31 (Fig. 2, bottom right panel). In the next section we analyze the pre-eruption filament evolution as seen in $\mathrm{H} \alpha$ high-resolution images by the Kanzelhöhe Solar Observatory, followed by the $3 \mathrm{D}$ reconstruction of the prominence eruption derived from STEREO data.

\section{Data analysis}

\subsection{Pre-eruption filament evolution}

The August 31, 2007, eruption occurred on the west limb, as seen from the Earth. Hence, it is possible to study in detail the 

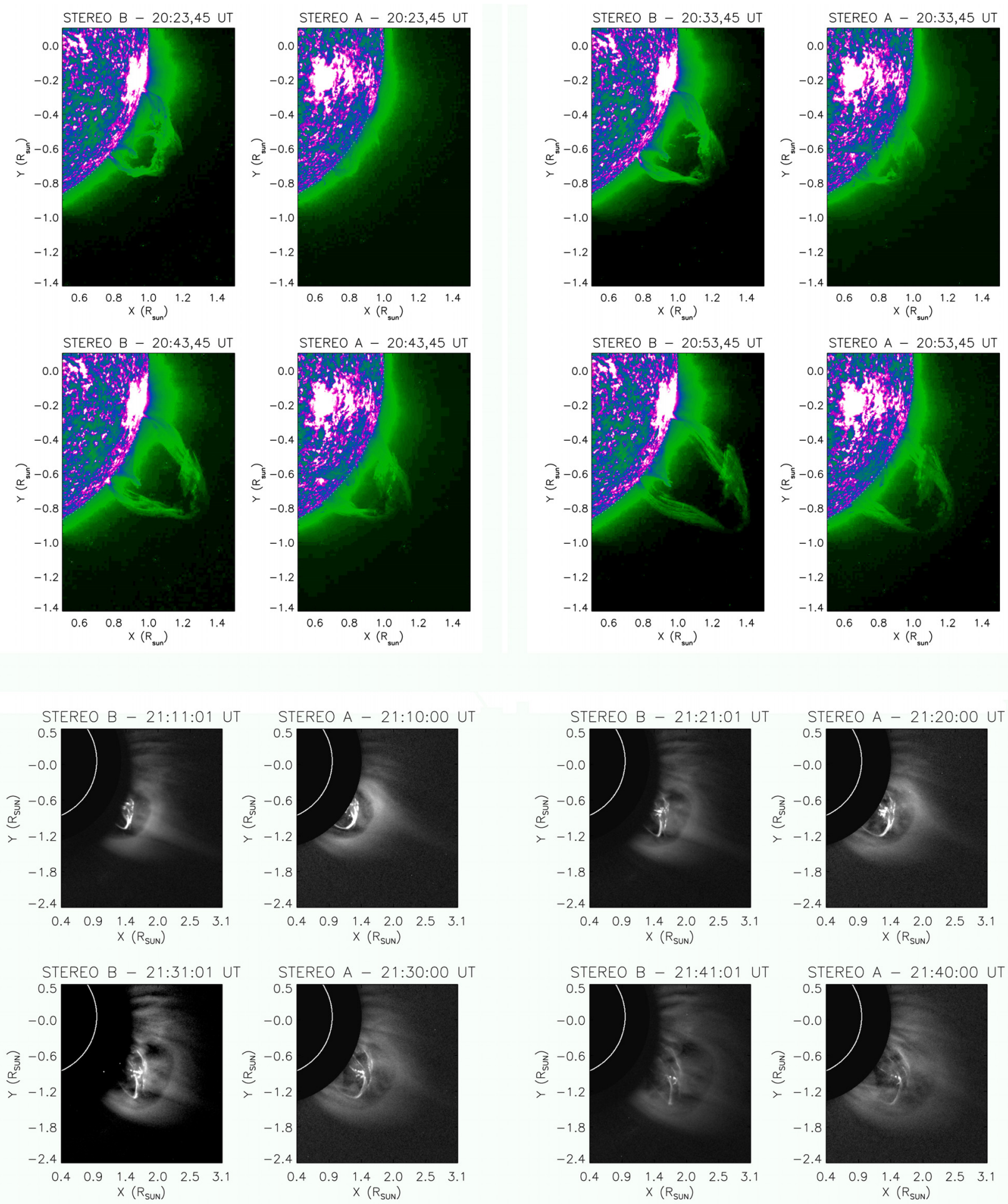

Fig. 1. Top 8 panels: the August 31, 2007, prominence eruption as seen by the EUVI instrument (HeII 304 filter) by the two STEREO-B (left) and STEREO-A (right) spacecraft at four different times. The color scale has been changed to better show the off-limb erupting plasma. Bottom 8 panels: the CME of August 31, 2007, as seen by the COR1 telescopes onboard STEREO-B (left) and STEREO-A (right) at four different times. The prominence observed at earlier times by EUVI (top) is associated in the COR1 images (bottom) to the "loop-like" feature visible in the expanding CME core.

pre-eruption evolution of the prominence in $\mathrm{H} \alpha$ images acquired by ground-based observatories during its transit from the east to the west limb following the solar rotation. Images acquired on August 22 (i.e., nine days before the eruption) by the Mauna Loa Observatory (Fig. 2, top left panel) show that both the Eand $\mathrm{W}$-branches have a clockwise inclination with respect to the meridional direction. Interestingly, $\mathrm{H} \alpha$ images acquired in the following days (Fig. 2, top right and bottom left panels) show a progressive counter-clockwise rotation of both branches: one week later both branches are mainly aligned with the meridional direction (Fig. 2, bottom panels). 


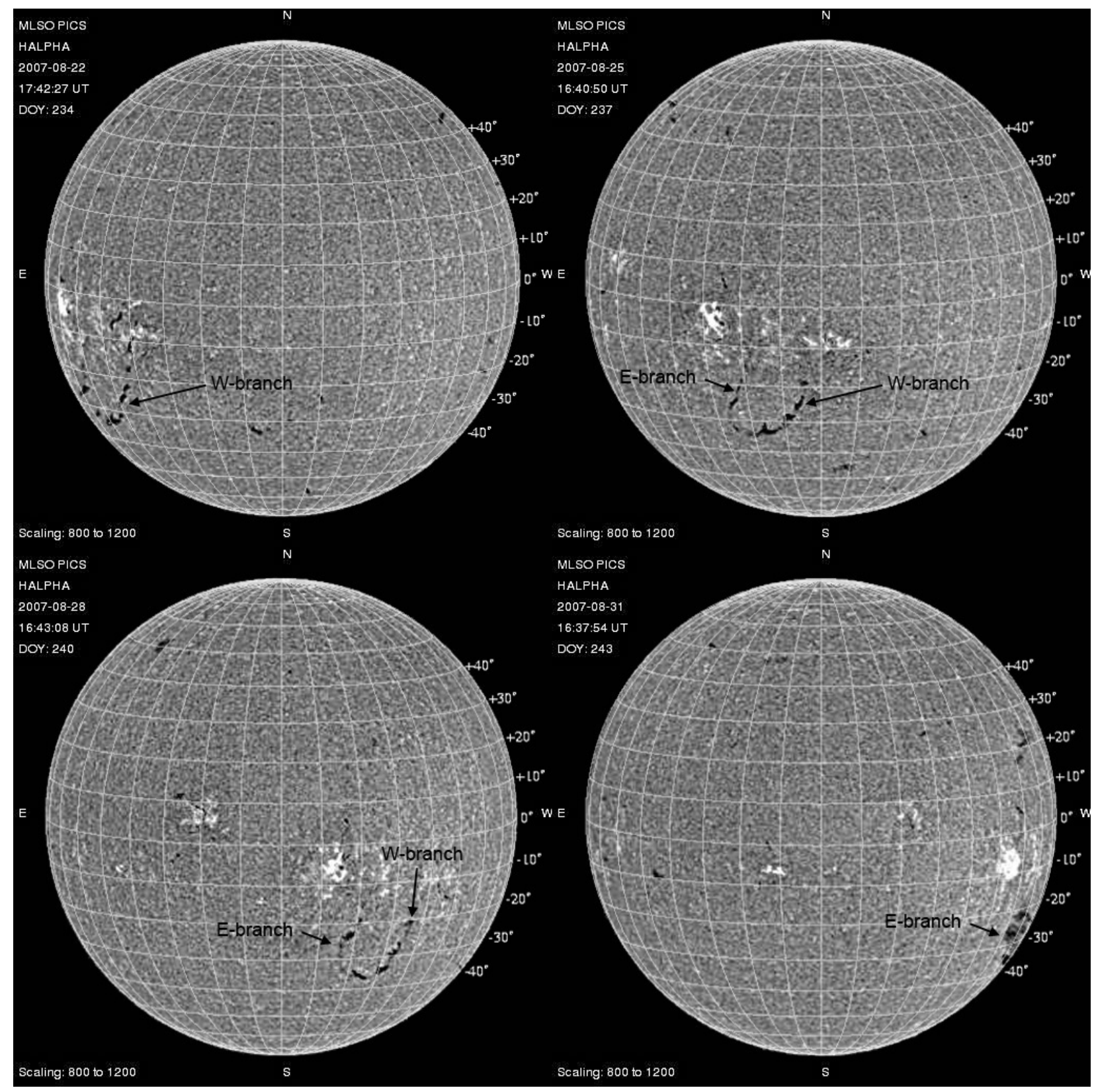

Fig. 2. Full disk H $\alpha$ images acquired on August 22 (17:42 UT, top left), 25 (16:40 UT, top right), 28 (16:43 UT, bottom left) and 31 (16:37 UT, bottom right) by the Mauna Loa Solar Observatory. This sequence shows that in the days before its eruption the filament is "U-shaped", with a westward and an eastward branch. Only the eastward and narrower part of the filament (the one visible close to the limb in the August 31 image) will be ejected starting from $\sim 19$ UT.

To provide a quantitative estimate of the observed preeruption filament rotation, we analyzed the high-resolution (1.09 arcsec/pixel) full disk $\mathrm{H} \alpha$ images acquired by the Kanzelhöhe Solar Observatory (Austria, see http://www . kso. ac.at/praesentation/praesentation_en.php). We first considered only the "upper" part (i.e., the one located at higher latitudes) of each branch and identified for each of the nine H- $\alpha$ images available between August 22-30 (Fig. 3) the positions of two E- and W-branch footpoints $P_{1}$ and $P_{2}$. In the following analysis, the latitude $\theta$ is the angle measured from the solar equator $\left(-90^{\circ} \leq \theta \leq+90^{\circ}\right)$, while the longitude $\phi$ is the angle measured from the central meridian as seen from Earth $\left(-180^{\circ} \leq \phi \leq+180^{\circ}\right)$. We computed the corresponding latitudes $\left(\theta_{1}, \theta_{2}\right)$ and longitudes $\left(\phi_{1}, \phi_{1}\right)$ for the points $P_{1}$ and $P_{2}$, by assuming that these points are located over the spherical solar surface. Given their latitudes and longitudes, we simply computed the length of the segment $s=\overline{P_{1} P_{2}}$ and the length of its projection along a meridian $l$ (by neglecting the solar surface curvature for simplicity) as

$$
\begin{aligned}
s= & R_{\odot}\left[\left(\cos \theta_{2} \sin \phi_{2}-\cos \theta_{1} \sin \phi_{1}\right)^{2}+\left(\cos \theta_{2} \cos \phi_{2}\right.\right. \\
& \left.\left.-\cos \theta_{1} \cos \phi_{1}\right)^{2}+\left(\sin \theta_{2}-\sin \theta_{1}\right)^{2}\right]^{1 / 2} \\
l= & R_{\odot}\left[\left(\cos \theta_{2} \sin \phi_{1}-\cos \theta_{1} \sin \phi_{1}\right)^{2}+\left(\cos \theta_{2} \cos \phi_{1}\right.\right. \\
& \left.\left.-\cos \theta_{1} \cos \phi_{1}\right)^{2}+\left(\sin \theta_{2}-\sin \theta_{1}\right)^{2}\right]^{1 / 2} .
\end{aligned}
$$

Given $s$ and $l$, the angle $\alpha$ between the filament branch and the meridional direction is simply given by $\alpha=\arccos (l / s)$.

From the above analysis we conclude that in the 9 days before the eruption (August 22-30, 2007) both filament branches rotated by $\sim 40^{\circ}$ in the counter-clockwise direction. This rotation cannot be simply ascribed to projection effects: as the Sun rotates from East to West, any dark filament lying over the solar surface 

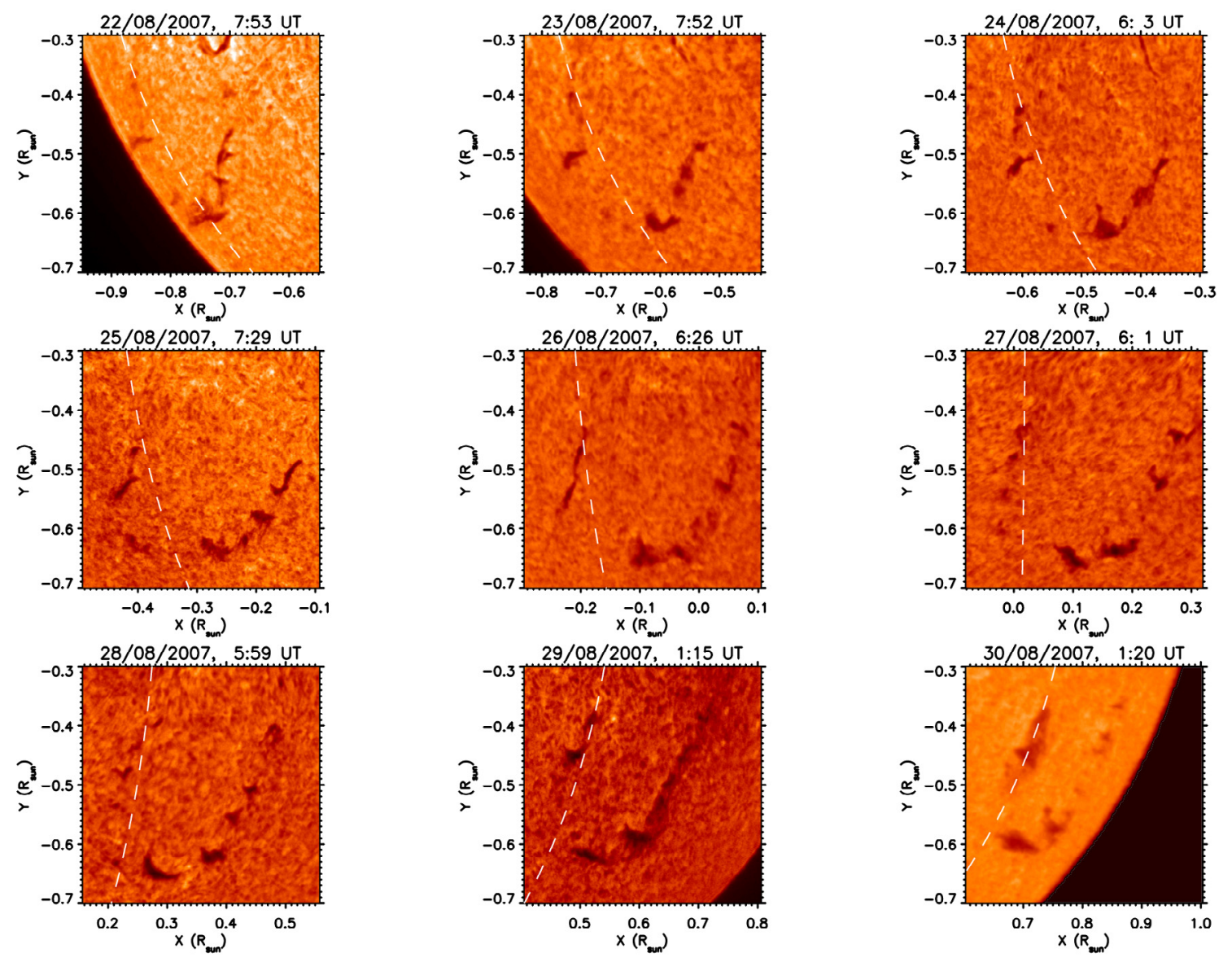

Fig. 3. Zoom on the filament branches as seen in $\mathrm{H} \alpha$ images acquired between August 22-29 by the Kanzelhöhe Solar Observatory and on August 30 by the Mauna Loa Observatory (no Kanzelhöhe image available from this day). This sequence shows the evolution of the two filament branches in the days before the eruption. Both filament branches show a strong clockwise inclination with respect to the meridian crossing it (dashed white line), one week before the eruption. This inclination progressively decreases with time, leaving both filament branches mostly aligned with the meridian one day before the eruption.

in the southern (northern) hemisphere is expected to show an apparent clockwise (counter-clockwise) rotation, so that for instance a filament aligned with the meridional direction close to the east-limb will be as well aligned with that direction $\sim 2$ weeks later at the west-limb. This is not the case for the two branches of the filament reported here, which (as Fig. 3 clearly shows), mainly keep a constant apparent inclination during their transit across the solar disk. This effect can be explained only by a counter-clockwise rotation of both filament branches.

$\mathrm{H} \alpha$ images apparently do not show any significant difference between the two branches that could explain why only the E-branch eventually erupted. Nevertheless, this is the filament branch closer to a small active region (NOAA 10969) located near the equator $\left(05^{\circ} \mathrm{S}-03^{\circ} \mathrm{E}\right.$ on August 27, 09:14 UT) and including a single small spot (of negative polarity) lying westward of a $\mathrm{H} \alpha$ facula (associated to a region of more disperse positive polarity), as we concluded from a comparison between $\mathrm{H} \alpha$ images and SOHO/MDI magnetograms (Fig. 4, top panels). Hence, it is likely that the presence of the small active region played a role in the final destabilization of the E-branch. Moreover, coronal fieldlines extrapolated with the potential field source surface approximation (PFSS) show that on August 24 (Fig. 4, bottom left panel) the filament lies below two systems of closed arcades: one lower altitude system (Fig. 4, bottom left panel, yellow lines), nicely bridging the magnetic neutral line (Fig. 4, top right panel, yellow line) and a higher altitude system, mostly aligned with the meridional direction. Interestingly,
PFSS field-lines extrapolated a few days later on August 28 show that the lower altitude system mainly lies mainly in the same position, while the higher altitude system is mostly aligned with the equatorial direction. Hence, apparently, before the eruption a rotation of higher altitude coronal fields overlying the filament occurred in the clockwise direction, i.e., in a direction opposite to the filament rotation observed in $\mathrm{H} \alpha$. Interestingly, the erupting filament underwent a rotation in the same sense as the preeruption overlying fieldlines, as we show in the next section from STEREO EUVI and COR1 data.

\section{2. $3 D$ reconstruction of the prominence eruption}

The filament described in previous section erupted on August 31. As mentioned, only the E-branch erupted, while the W-branch did not. The eruption of the filament was observed from both STEREO spacecraft, hence 3D coordinates of the prominence plasma can be determined via triangulation. The positions of the two spacecraft and the point in the solar corona to be triangulated define a plane called the epipolar plane (e.g., Inhester 2006). Because every epipolar plane is seen head-on from both spacecraft, it is reduced to a line in the respective image projections; this line is called epipolar line. Any object identified to be situated on a certain epipolar line in one image must lie on the same epipolar line in the other image. Finding a correspondence between pixels in the images taken by spacecraft 1 and 2 


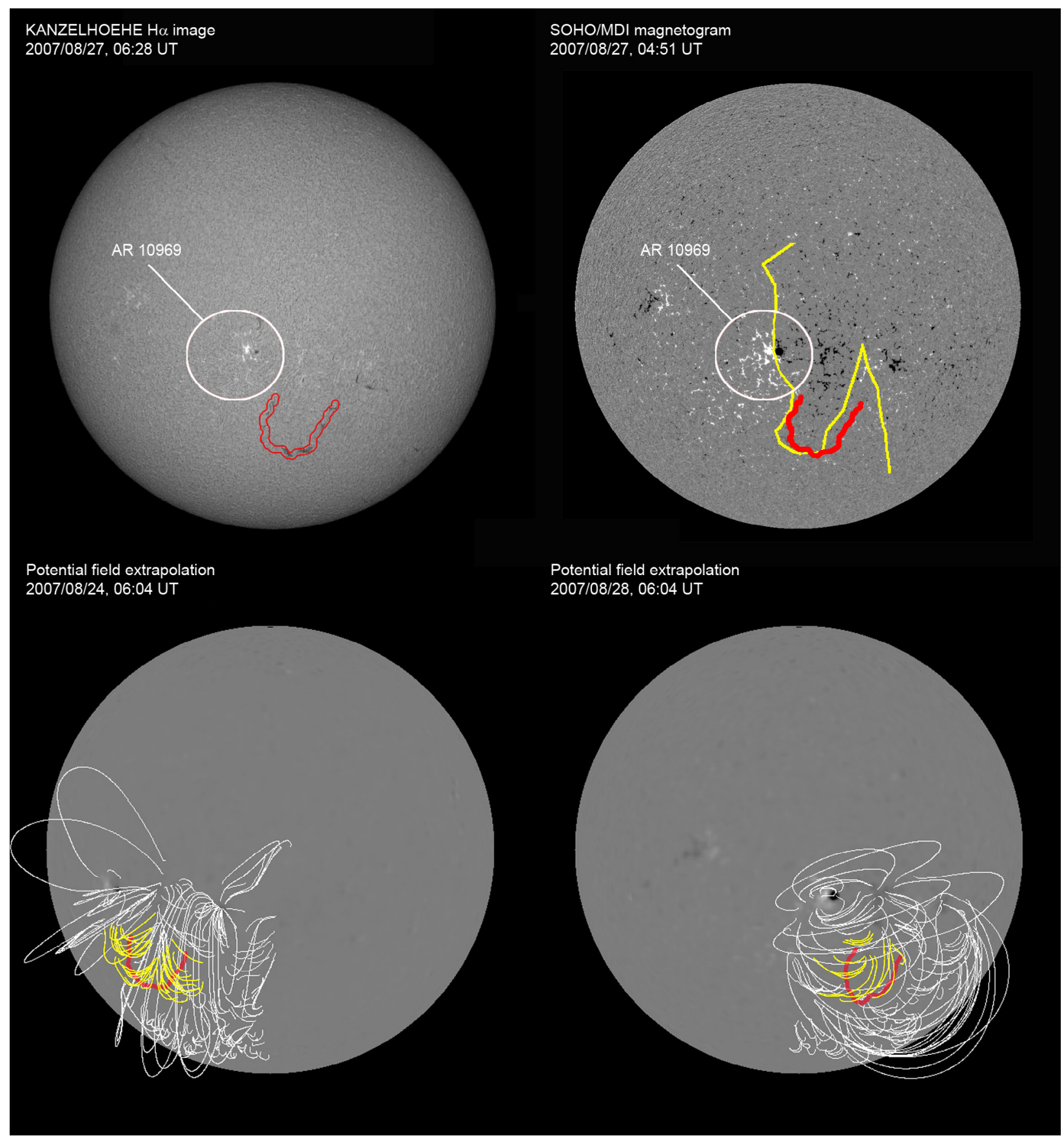

Fig. 4. Overall magnetic field configuration before the August 31 eruption. Top left: Kanzelhöhe H $\alpha$ image acquired on August 27 showing the relative position of the filament (surrounded by a red line) and the active region (white circle). Top right: SOHO/MDI photospheric magnetic field map on August 27 showing the position of the filament (red line) with respect to the different magnetic field polarities and to the magnetic neutral line (yellow line, from the Wilcox Solar Observatory). Bottom: potential field extrapolations as derived for August 24 (left) and August 28 (right) showing the position of closed fieldlines overlying the active region and the $\mathrm{H} \alpha$ filament (red line).

is therefore reduced to establish this correspondence along the same epipolar lines in both images. Once the corresponding feature is found in the two images, the $3 \mathrm{D}$ reconstruction is achieved by calculating the lines of sight that belong to the respective feature in the image and tracking them back into the 3D space. Because the lines of sight have to lie in the same epipolar plane, their intersection in this plane is unambiguously defined (e.g., Inhester 2006), allowing us to reconstruct the 3D coordinates of the selected point. This technique is usually referred to as "tie-pointing" technique. The program we used for this reconstruction (scc_measure.pro) is available in the Solar Software package. This routine was developed by W. T. Thompson and first applied by the developer to reconstruct the $3 \mathrm{D}$ orientation of sungrazing comet tails (see Thompson 2009).

For this study we use EUVI $304 \AA$ images, with a spatial resolution of 1.6 arcsec and a temporal resolution of $2.3 \mathrm{~min}$. To reconstruct the $3 \mathrm{D}$ shape of the filament at different times, we selected 13 frames acquired during the eruption by the STEREO/EUVI with the HeII 304 filter, starting from 20:23 UT untill 20:53 UT. The selection of this time interval is dictated by the following considerations: images acquired before 20:23 UT by STEREO-A show the erupting filament entirely on-disk (making the identification of HeII knots, filaments or threads harder), while images acquired after 20:53 UT by STEREO-B 
show that the filament is far out in the EUVI field of view and there are only a few counts at the top of the filament (see Fig. 1, top panels).

For each one of these frames we visually selected a series of pair points located along the filament (typically about 2530 points per frame) and, by using the $s c c$ measure.pro routine, we reconstructed the 3D shape of the filament at each time. For the first frames a large part of the filament is seen by STEREO-A on-disk, while the same fraction of the filament is observed at the same time by STEREO-B off-limb. Because of this, it was necessary for a few points to associate different parts of the filament seen in emission by STEREO-B (i.e., brighter than the background) with parts seen in absorption by STEREO-A (i.e., darker than the background), by assuming that the emitting/absorbing plasma is the same.

As the filament expands, the eruption results in a slow CME, which is also observed by both the STEREO/COR1 coronagraphs. Figure 1 (bottom panels) shows the eruption observed at four different times by COR1 onboard the two STEREO$A$ and -B spacecraft. Total brightness (tB) COR1 images were derived from three sequential images taken with polarization of 0,120 and 240 degrees. To remove the contribution of the streamers in COR1 images and visualize the contribution of the CME alone, we subtracted a minimum model image from each tB image. The minimum image is created by taking the minimum value in each pixel over the images of the day when the CME was observed. The sequence acquired by COR1 instruments clearly shows the typical CME three-part structure, with an opening bright front including a dark cavity that surrounds the expanding brighter prominence. Because the core of the CME (i.e., the filament) can be identified in both views, we applied the same tie-pointing technique to the COR1 data to reconstruct the 3D shape of the filament at later times. We selected seven frames recorded by COR1 between 21:15 UT and 21:45 UT on August 31st. Combining the data from EUVI and COR1, we reconstructed the 3D structure of the erupting prominence for $1 \mathrm{~h}$ and $22 \mathrm{~min}$.

An example of 3D filament reconstructions obtained from EUVI data is provided in Fig. 5 at three different times during the eruption. This figure shows the reconstructed filament as seen in a reference frame rotated to simulate its appearance from an observer placed on Earth (top left panel), on the ecliptic plane above the west limb (top right panel) and on the plane of sky (as seen from the Earth) above the north limb (bottom left panel). Moreover, to study the occurrence of filament rotations during the eruption, the reconstructed filament was also rotated by its central latitude $\left(\sim 25^{\circ} \mathrm{S}\right)$ and longitude $\left(\sim 66^{\circ} \mathrm{W}\right)$, to show the eruption as seen by an observer located above the eruption site (bottom right panel). Results from these reconstructions are described in the next section.

\section{Results}

\subsection{Prominence kinematics and rotation}

Given the 3D filament reconstruction at each time from the EUVI and COR1 instruments, it is possible to study in detail the kinematical parameters of the eruption. In particular, Fig. 6 shows the time evolution of the maximum altitude (top left panel), the average latitude (top right panel) and average longitude (bottom left panel) of the filament. In each plot we show the data points from EUVI (solid red line) and COR1 (solid blue line), together with analytic curves resulting from simple polynomial fitting (dashed lines). Uncertainties on these parameters were empirically estimated by selecting a range of different pairpoints with tie-pointing technique that are possibly associated with the same He II feature.

These plots show that during the slow expansion $(v \sim$ $150 \mathrm{~km} \mathrm{~s}^{-1}$ ) the filament underwent a small acceleration in the radial direction $\left(a \simeq 11.8 \mathrm{~m} \mathrm{~s}^{-2}\right)$ and that significant variations of the filament latitude and longitude also occurred. The latter ones are not only the consequence of a non-radial expansion. Indeed, if we for instance consider the simple case of a prominence expanding at constant longitude $\phi$ with any law $s=s(t)$ along a linear trajectory $s$ inclined in latitudinal direction with an angle $\beta$ with respect to the radial and leaving from the initial latitude $\theta_{0}$, then the law $\theta=\theta(t)$ providing the dependence of the prominence latitude $\theta$ as a function of time is simply

$\theta(t)=\theta_{0}+\arctan \frac{s(t) \sin \beta}{s(t) \cos \beta+s_{0}}$,

with $s_{0}=1 R_{\odot}$. A similar curve also holds for the longitudinal variations $\phi=\phi(t)$. If we simply assume $s=s(t)$ as being the one from the polynomial fit of the altitude vs time curve (Fig. 6, top left panel), the curves for $\theta(t)$ and $\phi(t)$ expected for linear propagation are shown in Fig. 6 with dotted lines (top right and bottom left panels). A comparison between the dotted curves and the observed values clearly shows that significant deflections with respect to a linear non-radial propagation occurred in both the latitudinal and longitudinal directions, also at higher altitudes. In particular, the longitudinal deflection is also visible in panels $\mathrm{c}$ and $\mathrm{g}$ of Fig. 5.

From the $3 \mathrm{D}$ structure of the filament at different times it is also possible to determine the evolution of its rotation angle during the eruption. To this end, we applied the following technique: at each time we determined the point of maximum altitude $h_{\max }$ (i.e., the filament top). Then, we identified the positions of the two points located at the same altitude $f h_{\max }$ along the filament, where $0<f<1$ is a fraction of the maximum altitude. These two points define a vector $\boldsymbol{v}_{f h}$ at the altitude $f h_{\max }$ (see Fig. 7). At the same time, the filament footpoints, visible in the EUVI data, define a "base" vector $\boldsymbol{v}_{\mathrm{b}}$. The angle $\alpha_{f h}$ between these two vectors gives an estimate of the filament rotation at the altitude $f h_{\max }$ with respect to the filament footpoints (see Fig. 7). In particular we selected $f=4 / 5$ for EUVI data and $f=14 / 15$ for COR 1 data, to study only the rotation of the higher part of the filament. The positions of the filament footpoints are not available for COR1 data; nevertheless, because EUVI data show that the two footpoints are in first approximation aligned with a meridional direction (see also Fig. 5, panel d), the angles from COR1 data were measured simply between the vector $\boldsymbol{v}_{f h}$ and the meridional direction.

The resulting evolution of the rotation angle $\alpha_{f h}$ as a function of time is shown in the bottom right panel of Fig. 6 as derived from the EUVI (red solid line) and COR1 (blue solid line) instruments, together with a polynomial fitting (dashed line). Error bars in this plot represent the typical variation of the measured rotation angle obtained with a variation of the fraction $f$ by $10 \%$. The plot clearly shows that a significant rotation of the filament occurred during its eruption. In particular, we conclude from the EUVI data that the filament part closer to the top rotated by nearly $50^{\circ}$ clockwise during the first $30 \mathrm{~min}$ of the eruption. Later on, we see from the COR1 data that a filament clockwise rotation is still occurring at least up to $\sim 1.5 \mathrm{~h}$ after the eruption start time, even if at a smaller rate. Hence, the EUVI and COR1 data lead us to conclude that the filament rotated clockwise by $\sim 90^{\circ}$ during the eruption. To better determine the consequences of this rotation, we performed a simple 

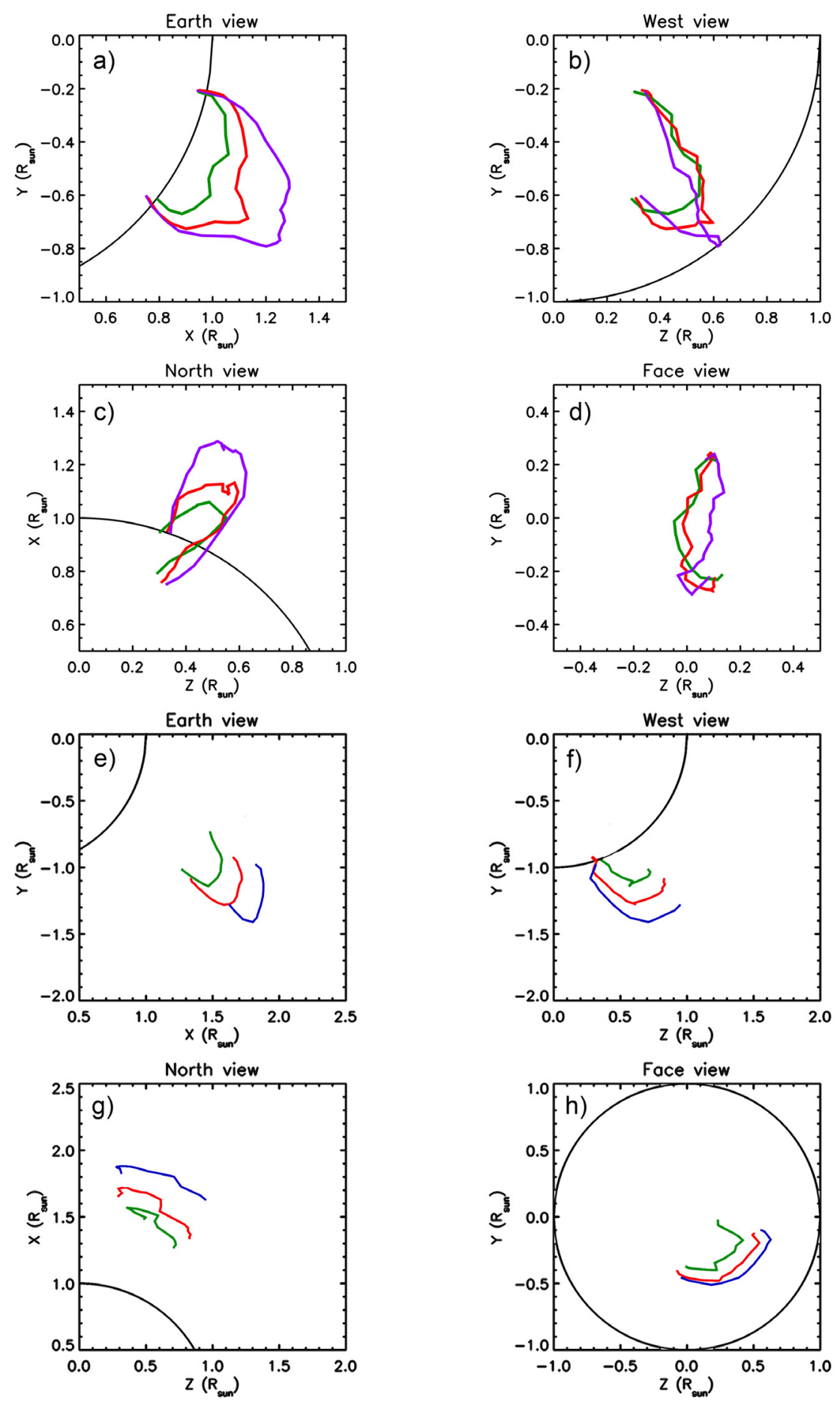

Fig. 5. Panels a-d) sequence of 3D prominence reconstructions as obtained from EUVI data acquired at 20:18 UT (green lines), 20:33 UT (red lines) and 20:46 UT (blue lines). The 3D reconstructions are shown as seen by an observer placed on Earth (XoY plane, panel a)), on the ecliptic plane above the west limb ( $Z o Y$ plane, panel b)), on the plane of sky above the north limb (ZoX plane, panel c)), and above the eruption site (panel d)). The $X Y Z$ axes are those of the standard heliographic coordinate system, having the origin $o$ at the Sun center and the $X$-, $Y$-, and $Z$-axes pointing toward the west limb, the north limb, and the Earth, respectively. Panels e-h) sequence of 3D prominence reconstructions as obtained from COR1 data acquired at 21:15 UT (green lines), 21:30 UT (red lines) and 21:45 UT (blue lines). The 3D reconstructions are shown as seen from the same reference systems as those employed for the reconstructions resulting from EUVI data.

linear fit of the $\alpha_{f h}$ vs. time curve (bottom right panel of Fig. 6). This showed that the filament rotated at an average angular velocity $\left\langle\dot{\alpha}_{f h}\right\rangle=5.26 \times 10^{-4} \operatorname{rad~s}^{-1}\left(=108 \mathrm{deg} \mathrm{h}^{-1}\right)$. Second order polynomial fitting provides a negative acceleration of the rotation angle equal to $\ddot{\alpha}_{f h}=-1.28 \times 10^{-7} \mathrm{rad} \mathrm{s}^{-2}$, with an initial rotation velocity $\dot{\alpha}_{f h 0}=6.04 \times 10^{-4} \mathrm{rad} \mathrm{s}^{-1}$.
The clockwise filament rotation observed during the eruption with He II $304 \AA$ data can now be compared with the counterclockwise rotation observed in $\mathrm{H} \alpha$ images for the E-branch in the days before the eruption. In particular, the resulting global evolution of the rotation angle observed before and during its eruption is shown Fig. 8. The jump in the right part of the plot is 

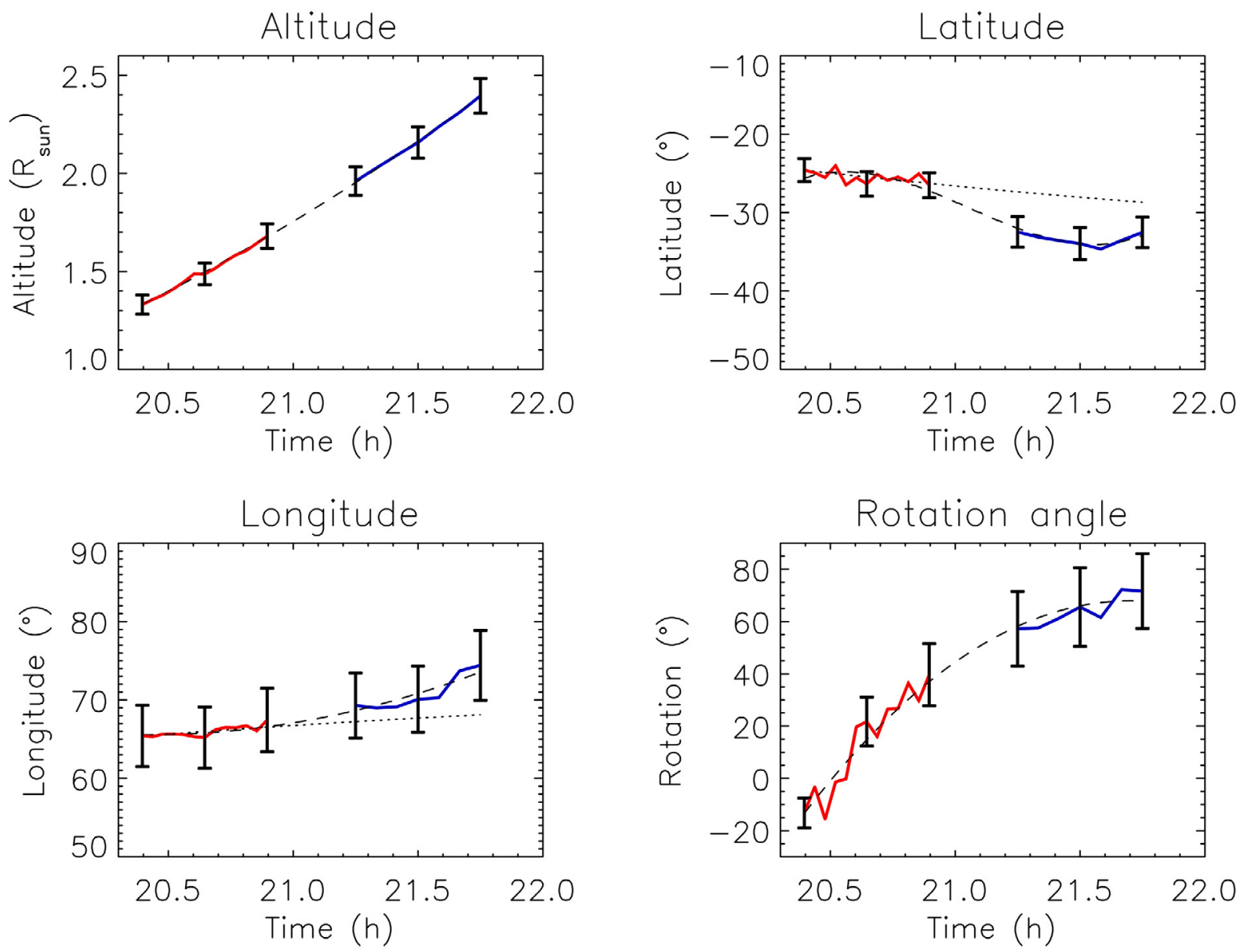

Fig. 6. Time evolution of the prominence top altitude (top left panel), average latitude (top right), average longitude (bottom left) and rotation angle (bottom right) as derived from EUVI (red solid lines) and COR1 (blue solid lines) data. Dashed lines in each panel refer to second order polynomial fits to the observed curves. Dotted lines show the latitudinal and longitudinal trends expected for an eruption expanding along a linear trajectory not aligned with the radial direction.

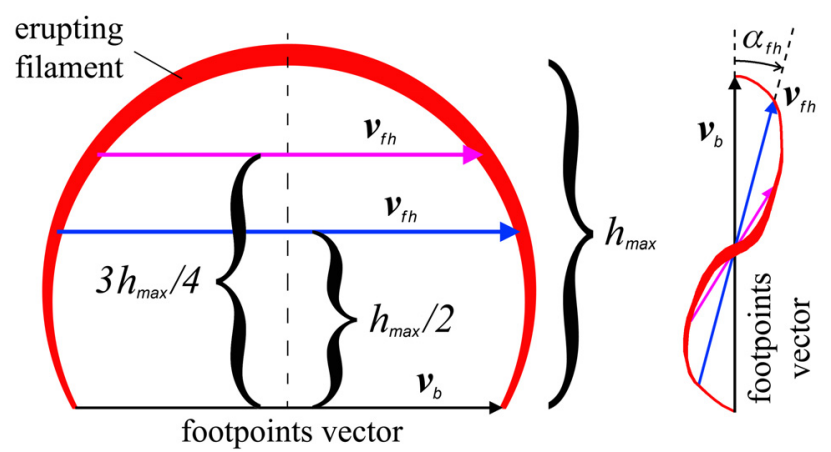

Fig. 7. Cartoon showing an example of two vectors $\boldsymbol{v}_{f h}$ located at fractions $f=1 / 2$ (blue) and $f=3 / 4$ (purple) of the maximum filament altitude $h_{\max }$ and the "base" vector $\boldsymbol{v}_{\mathrm{b}}$ connecting the filament footpoints (black). The left plot shows the filament seen off-limb as a prominence, while the right plot shows the filament as seen from an observer located above the filament. If the higher part of the filament is rotated with respect to the position of the two footpoints, then the rotation angle $\alpha_{f h}$ can be determined as the angle between the vectors $\boldsymbol{v}_{f h}$ and $\boldsymbol{v}_{\mathrm{b}}$.

the rotation observed from EUVI data during the eruption (see previous plots). This figure shows that in the days before the eruption the filament underwent a very slow counter-clockwise rotation in the opposite direction with respect to the rotation

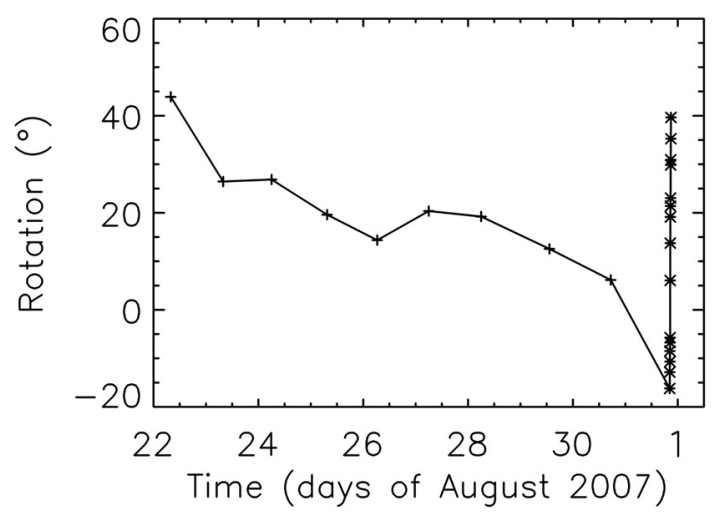

Fig. 8. Evolution with time of the inclination angle with respect to the meridional direction for the the E-branch of the filament in the days before the eruption. The left part of the curve shows the slow counterclockwise rotation observed in $\mathrm{H} \alpha$ images (see also Figs. 2 and 3) followed by the rapid clockwise rotation during the prominence eruption.

observed during the eruption both from STEREO/EUVI and COR1 data (latter part of the curve in Fig. 8). This interesting result will be discussed together with previous results in the last section. Please notice that in general, $\mathrm{He}$ II and $\mathrm{H} \alpha$ prominences 

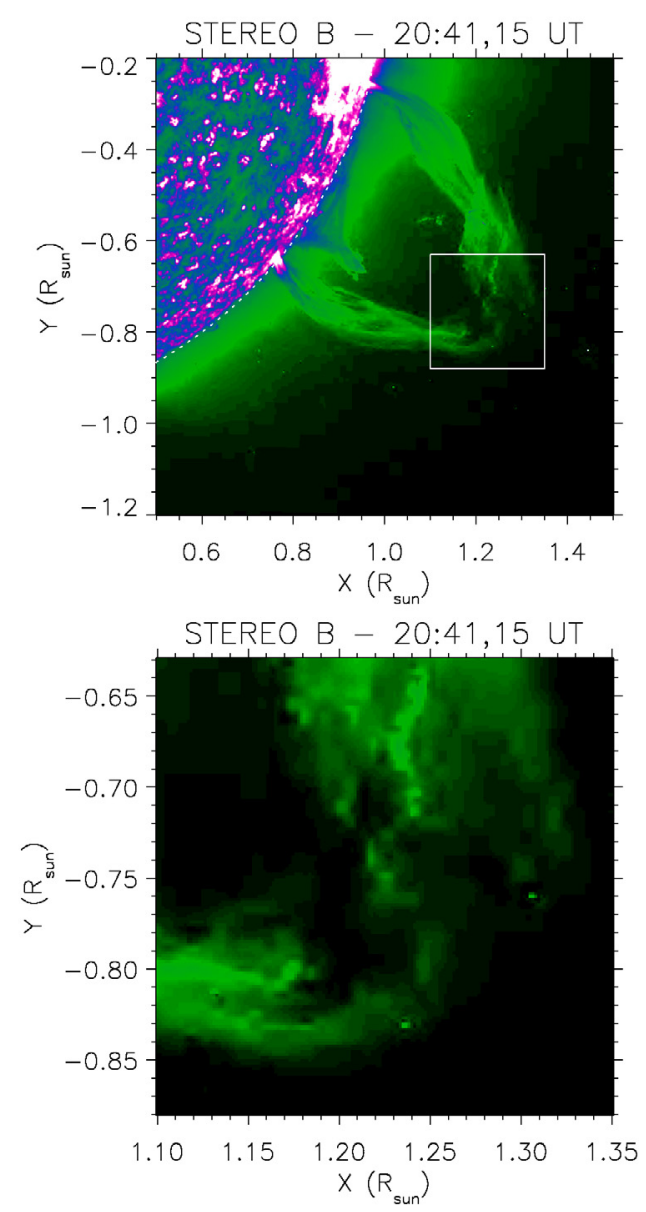
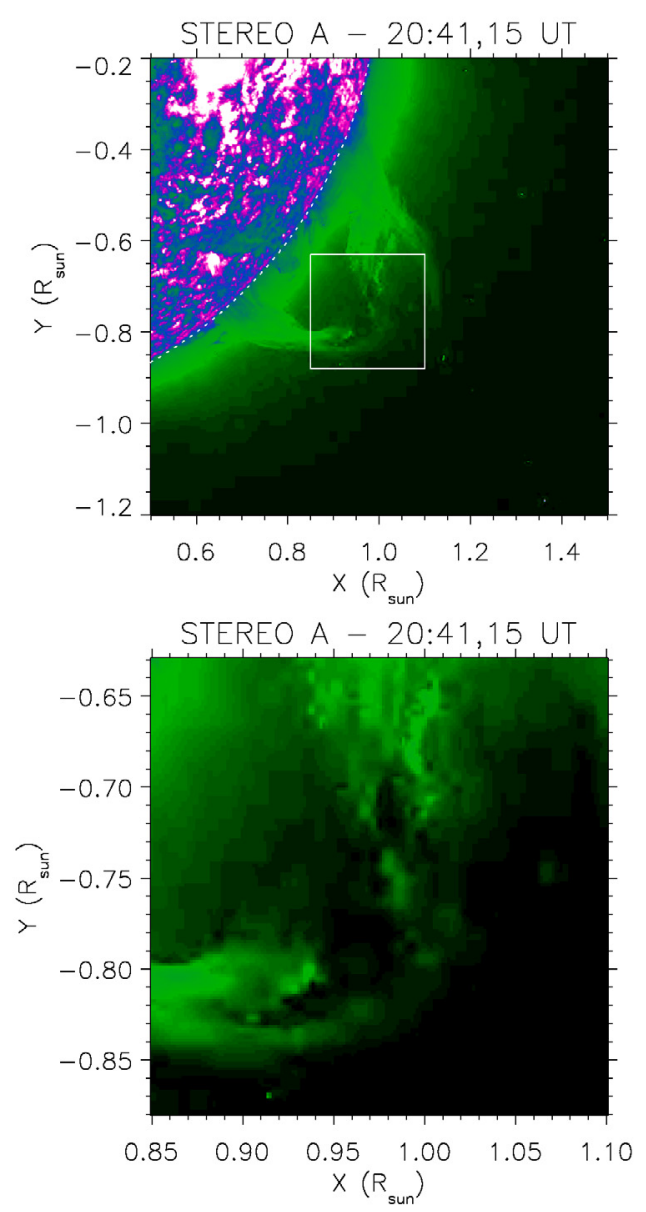

Fig. 9. Top: EUVI frames acquired by STEREO-B (left) and -A (right) at 20:41 UT when the small-scale helical feature at the top of the prominence was reconstructed. Bottom: a zoom on the small scale feature in the smaller detector area outlined in the top panels with white boxes.

do not have the same aspect as filaments when crossing the limb (e.g., Schmieder et al. 2003; Schwartz et al. 2006; Labrosse et al. 2010; Mackay et al. 2010). In any case, this does not affect our measurements, because both emissions outline the orientation of the same filament channel.

\subsection{Small-scale features}

Thanks to the high spatial resolution of the EUVI images $\left(\sim 1^{\prime \prime} 6\right.$ pixel $\left.^{-1}\right)$, we were able to determine the 3D structure of small-scale features present in the prominence as well. In particular, a closer inspection of the HeII images demonstrates that the filament itself contains a helical feature in its interior, which is clearly shown in Fig. 9. By again applying the tie-pointing technique to this helical feature, it is possible to determine the 3D position and 3D shape of this helix. To this end, we selected 41 pair points along the helix observed in the frame acquired at 20:41 UT. The resulting helix is quite complex, in that neither the radius, nor the periodicity of the helix are constant; hence, the analytical curve requires 11 free parameters to be fully described. In particular, we fitted the observed helix with the following curve:

$$
\begin{aligned}
& x^{\prime}(t)=x_{0}+\left(r_{0}+v_{r} t\right) \cos (\omega t+\phi) \\
& y^{\prime}(t)=y_{0}+\left(r_{0}+v_{r} t\right) \sin (\omega t+\phi) \\
& z^{\prime}(t)=z_{0}+v_{z} t+1 / 2 a_{z} t^{2}
\end{aligned}
$$

written as a function of a free parameter $t$ in a reference system $x^{\prime} y^{\prime} z^{\prime}$, which was then rotated by angles $\left(\alpha_{X}, \alpha_{Y}\right)$ with respect to the standard heliographic coordinate system $X Y Z$, having the origin $\mathrm{O}$ at the Sun center and the $X$-, $Y$-, and $Z$-axes pointing toward the west limb, the north limb, and the Earth, respectively. The helix is left- (right-)handed if the $\omega$ parameter is negative (positive).

The reconstructed 3D helix (red line) and a 3D fitting curve (blue line) of the observed points are shown in Fig. 10. From the curve fitting we conclude that the observed feature can be well approximated with a left-handed helix $(\omega<0)$ with radius $R=$ $r_{0}+v_{r} t$ linearly changing along its axis from $R=1.5 \times 10^{3} \mathrm{~km}$ to $r=8.0 \times 10^{3} \mathrm{~km} \simeq 0.011 R_{\odot} ; 2.3$ full rotations are required to describe the whole observed helix, whose extension in the direction parallel to its axis is of about $9.0 \times 10^{4} \mathrm{~km} \simeq 0.13 R_{\odot}$. Hence, a helical topology of the magnetic field exists at small spatial scales $\left(\sim 0.01-0.1 R_{\odot}\right)$ inside the erupting prominence.

\section{Discussion and conclusions}

On August 31, 2007, a prominence eruption was observed at the west limb by the STEREO/EUVI instrument, resulting in the core of a three part structure CME observed later on by the STEREO/COR1 telescope. We employed STEREO data to study the eruption kinematics and to provide information on the prominence rotation in the early phase of the eruption between heliocentric distances of $\sim 1.3$ and $\sim 2.4 R_{\odot}$. These data 


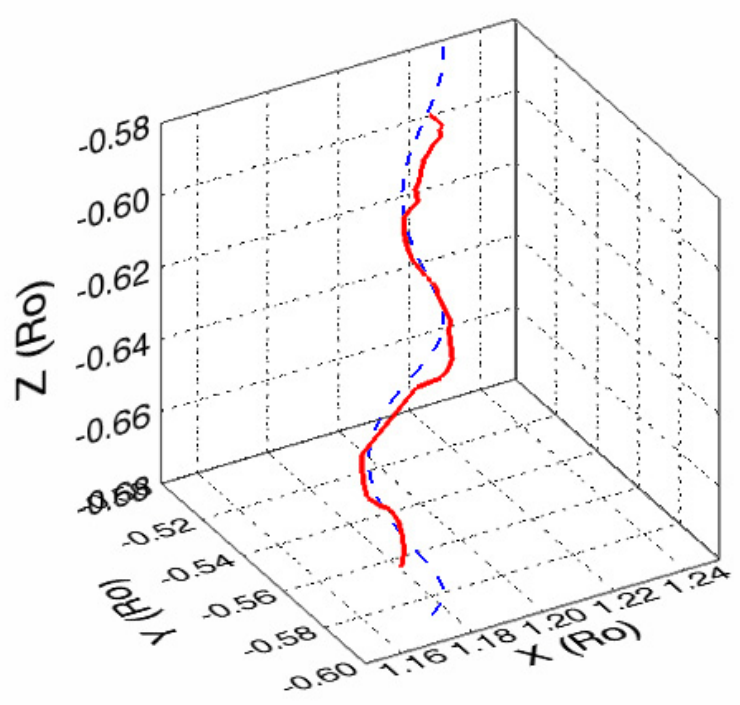

Fig. 10. Reconstructed 3D structure (solid red line) of the small-scale helical feature shown in Fig. 8 and the best-fit helical curve (blue dashed line).

were complemented with $\mathrm{H} \alpha$ images acquired from Earth-based observatories to provide information on the evolution of the preeruption filament. We summarize the main observational facts:

- H $\alpha$ images show an U-shaped filament, but only the eastward branch, closer to an active region, eventually erupts;

- the erupting branch rotates by $\sim 40^{\circ}$ counter-clockwise in the week before the eruption;

- during the eruption the prominence rotates clockwise by $\sim 90^{\circ}$; the rotation occurs with average angular velocity $\omega=$ $5.26 \times 10^{-4} \mathrm{rad} \mathrm{s}^{-1} \simeq 110 \mathrm{deg} \mathrm{h}^{-1}$;

- the rotation angular velocity decreases with time;

- the rotation of the erupting filament occurs in the opposite direction with respect to the pre-eruption $\mathrm{H} \alpha$ filament rotation and in the same direction as the apparent rotation of the overlying extrapolated potential field-lines;

- during the eruption, a net acceleration in the radial direction is present even in the later eruption phases; significant changes in the average filament latitude and longitude of propagation occur at intermediate altitudes;

- during the eruption, formation of a small-scale left-handed helical topology is observed at the top of the erupting prominence.

The observed rotation velocity decrease with time seems to indicate that some angular momentum has been initially provided to the prominence in the early phase of the eruption, while during the following expansion the prominence is loosing its angular momentum. Interestingly, the derived rotation velocity has a quite high value (about one order of magnitude higher) if compared with the average CME rotation rate recently determined by Yurchyshyn et al. (2009) from the analysis of SOHO/LASCO$\mathrm{C} 3$ data, hence at much higher altitudes. This agrees with the deceleration in the prominence angular rotation velocity reported here. The observed latitudinal and longitudinal deflection also indicates that some "tangential" forces (likely related to the structure of the magnetic field overlying and surrounding the erupting prominence) are also playing a role at intermediate altitudes, but are negligible at lower altitudes. Recently, Panasenco et al. (2010) found evidence of sideways rolling motions of erupting prominences and interpreted this effect as a consequence of force imbalances inside the filament arcade related to the adjacent large coronal holes, while the observed non-radial motions of the resulting $\mathrm{CME}$ are interpreted as a signature of global magnetic configuration force imbalances.

The most interesting result we obtained is the counterclockwise rotation of the filament and the clockwise rotation of the overlying magnetic fieldlines observed before the eruption, to be compared with the clockwise rotation of the filament during its eruption. These could be the signature of a magnetic helicity storage occurring not in the filament itself (as usually envisaged for instance in the helical-kink instability model), but in the global magnetic field configuration of the surrounding corona. This helicity (in analogy with what could happens for a spring that is compressed and released) is eventually released in a few hours during the eruption as a clockwise rotation of the rising filament. The effects of changes in a potential field overlying erupting filaments destabilized by kink- and torus instabilities have recently been described for instance by Liu (2008).

This interpretation would explain why there is no evidence for a sigmoidal structure of the filament in the days before the eruption, even if some amount of helicity has likely been accumulated also in the filament itself, as demonstrated by the observed small-scale helical feature at the top of the erupting prominence. This result agrees for instance with results derived by Muglach et al. (2009), who found in two events that the rotation of the filament axis is determined by the net helicity of the erupting system and that the surrounding field provides the main contribution to the net helicity. Lynch et al. (2009b) recently argued from breakout CME simulations that "observations of filament rotation and/or axial writhe occurring during or after the onset of flare reconnection are not sufficient to prove that the pre-eruption magnetic field configuration was that of a twisted flux rope in the corona". Also, there is mounting evidence that reconnections occurring between the expanding CME and the surrounding magnetic field may play a major role in the CME early evolution, as recently discussed and simulated by Bemporad et al. (2010). In particular, 3D simulations by Cohen et al. (2010) demonstrate that these reconnections may induce a rotation of the flux rope by $90^{\circ}$, which is not related to the kink instability.

The formation of a small-scale helical feature is observed at the top of the erupting prominence; EUVI images show that this feature progressively forms as the prominence expands. It is very interesting that the observed helix is left-handed, which means that its sense of rotation agrees with the observed large-scale clockwise rotation of the erupting prominence. Hence, these observations give a strong support to the conversion of twist into writhe of the same sign in a kink-unstable magnetic flux-rope, as reported for instance by Rust \& Labonte (2005); Zhou et al. (2006).

The $\sim 90^{\circ}$ rotation reported here and ascribed to the global coronal field configuration may also have a strong impact on the determination of the geo-effectiveness of the resulting interplanetary CME. Out-of-the-ecliptic components of the magnetic field dragged by the expanding flux rope may reconnect with the Earth magnetosphere and produce a geomagnetic storm. Nevertheless, the orientation of this component is determined by the orientation of the flux rope. If the observed rotation by $\sim 90^{\circ}$ of the prominence can be interpreted as an equivalent rotation of the expanding flux rope, similar rotations may have in general a strong impact on the definition of geoeffective CMEs, hence on the space weather forecasting. 


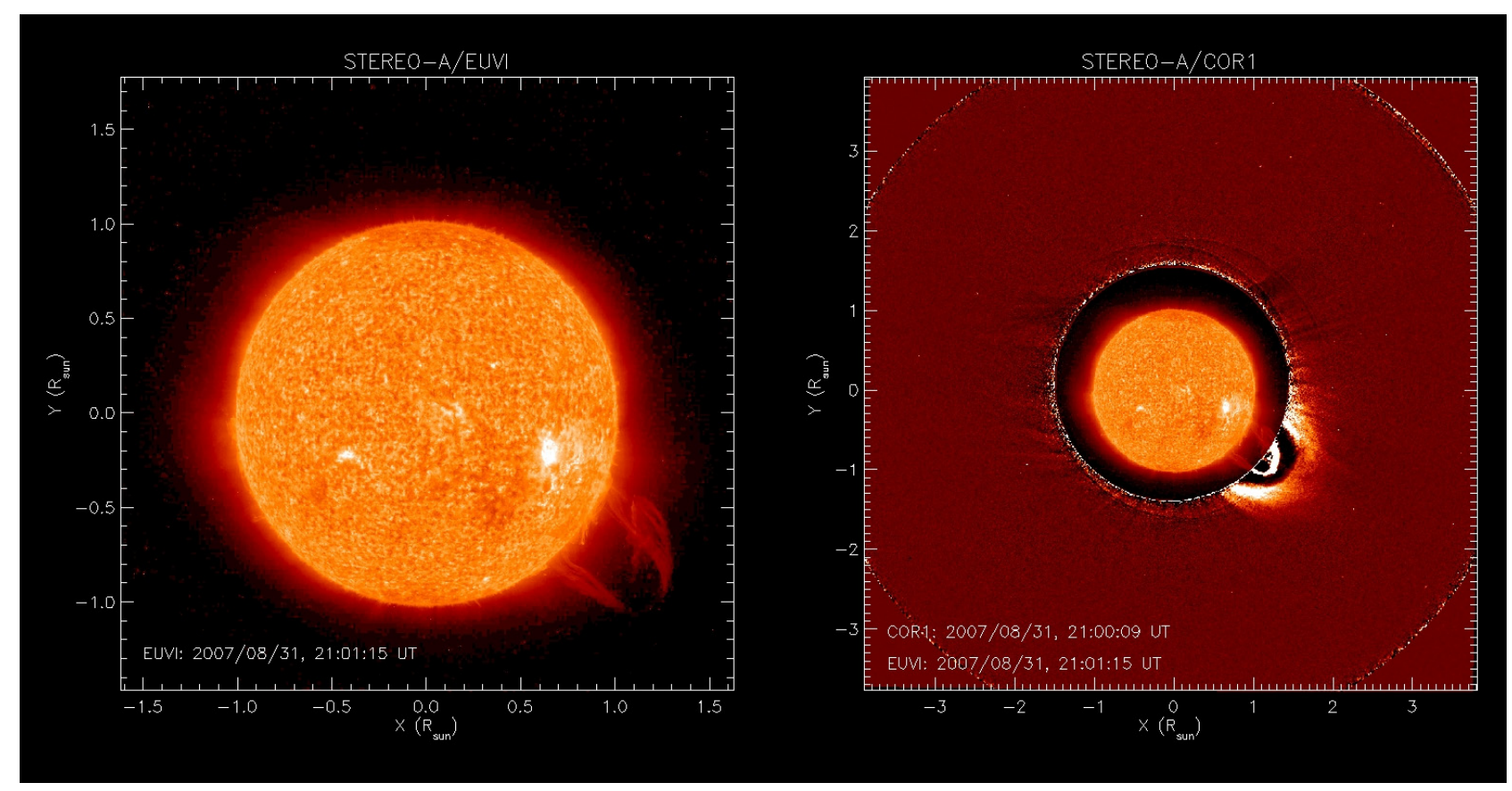

Fig. A.1. Snapshot from the STEREO-A movie available online. Left: EUVI He Ir $304 \AA$ image acquired by STEREO-A on August 31, 2007, 21:01:15 UT showing the prominence eruption on the southwest limb. Right: superposition between the same EUVI frame shown on the left and the COR1 image acquired at 21:00:09 UT. Each COR1 image in the movie is a base difference between the actual frame and the one acquired at 19:55:09 UT on the same day.

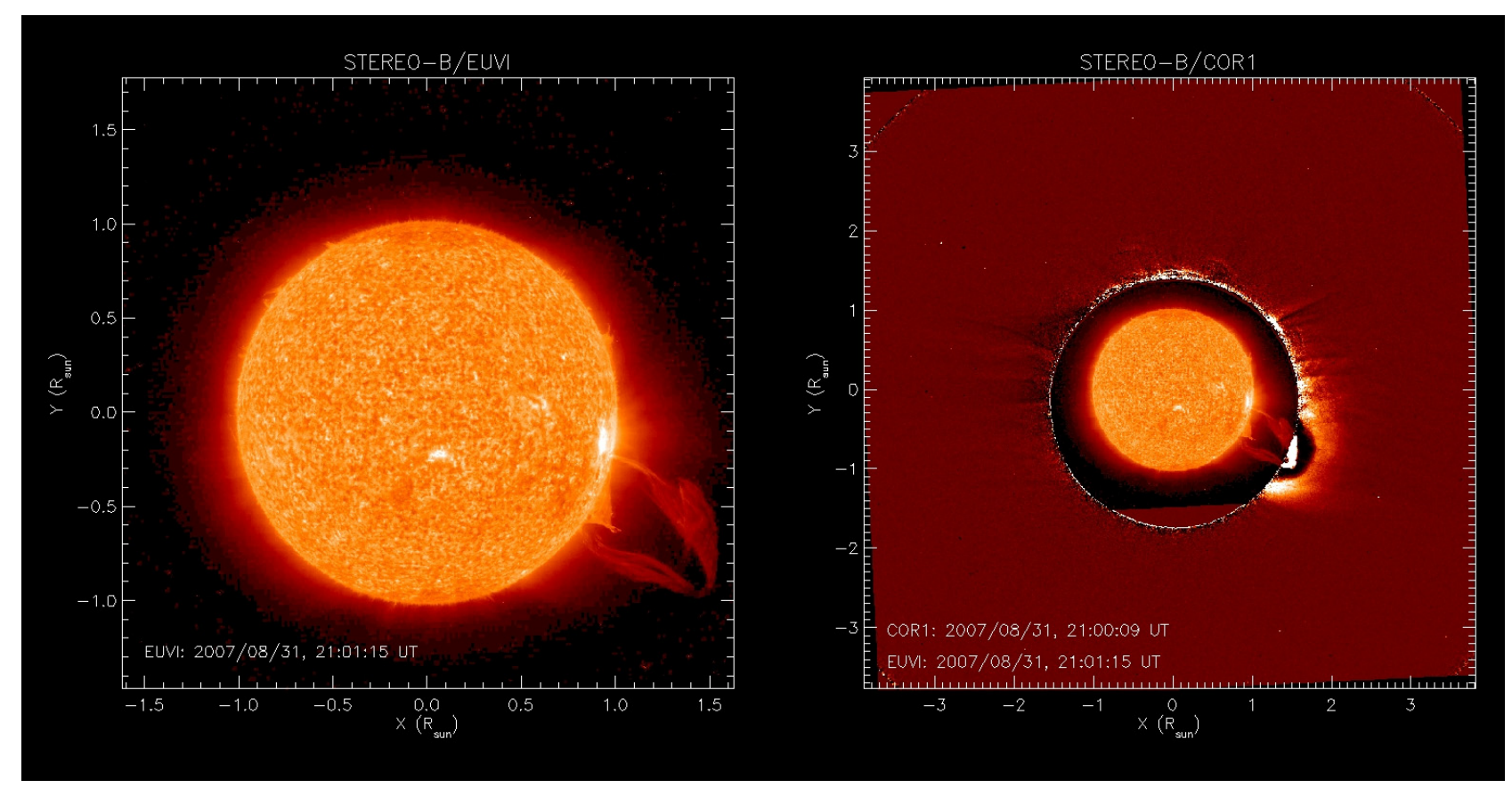

Fig. A.2. The same as in the previous figure, but for STEREO-B.

\section{Appendix A: Snapshots from the online movies}

This Appendix shows two snapshots from the STEREO-A and -B movies available on-line.

Acknowledgements. A.B. acknowledges support from ASI/INAF I/023/09/0 contract. STEREO is a NASA mission. D.T. acknowledges support from STFC.

\section{References}

Antonucci, E., Kohl, J. L., Noci, G., et al. 1997, Sol. Phys., 182, 293 Athay, R. G., Querfeld, C. W., Smartt, R. N., et al. 1983, Sol. Phys., 89, 3 Bemporad, A., Soenen, A., Jacobs, C., et al. 2010, Astrophys. J., 718, 251 Berger, M. A., \& Field, G. B. 1984, J. Fluid Mech., 147, 133

Canfield, R. C., Hudson, H. S., \& McKenzie, D. E. 1999, Geophys. Res. Lett., 26,627

Ciaravella, A., Raymond, J. C., Thompson, B. J., et al. 2000, ApJ, 529, 575 
A. Bemporad et al.: Rotation of an erupting filament

Cohen, O., Attrill, G. D. R., Schwadron, N. A., et al. 2010, J. Geophys. Res., 115, A 10104

Fan, Y., \& Gibson, S. 2004, ApJ, 609, 1123

Green, L. M., Kliem, B., Török, T., et al. 2007, Sol. Phys., 246, 365

Gopalswamy, N., Shimojo, M., Lu, W., et al. 2003, ApJ, 586, 562

Hood, A. W., \& Priest, E. R. 1981, Geophys. Astrophys. Fluid Dyn., 17, 297

Howard, R. A., Moses, J., Vourlidas, A., et al. 2008, Space Sci. Rev., 136, 67 Inhester, B. 2006 [arXiv: astro-ph/0612649]

Kaiser, M. L., Kucera, T. A., Davila, J. M., et al. 2008, Space Sci. Rev., 136, 5

Kohl, J. L., Esser, R., Gardner, L. D., et al. 1995, Sol. Phys., 162, 313

Kurokawa, H., Hanaoka, Y., Shibata, K., \& Uchida, Y. 1987, Sol. Phys., 108, 251

Labrosse, N., Heinzel, P., Vial, J.-C., et al. 2010, Space Sci. Rev., 151, 243

Liu, Y. 2008, ApJ, 679, L151

Lynch, B. J., Li, Y., Thernisien, A. F. R., et al. 2009a, J. Geophys. Res., 115, A07106

Lynch, B. J., Antiochos, S. K., Li, Y., et al. 2009b, ApJ, 697, 1918

Mackay, D. H., Karpen, J. T., Ballester, J. L., et al. 2010, Space Sci. Rev., 151, 333
Martin, S. F. 1998, Sol. Phys., 182, 107

Muglach, K., Wang, Y.-M., \& Kliem, B. 2009, ApJ, 703, 976

Noci, G., Kohl, J. L., \& Withbroe, G. L. 1987, ApJ, 315, 706

Panasenco, O., Martin, S., Joshi, A. D., \& Srivastava, N. 2010, J. Atmos. SolarTerrestr. Phys., in press

Rust, D. M., \& Kumar, A. 1984, Sol. Phys., 155, 69

Rust, D. M., \& Labonte, B. J. 2005, ApJ, 622, L69

Schmieder, B., Tziotziou, K., \& Heinzel, P. 2003, A\&A, 401, 361

Schwartz, P., Heinzel, P., \& Schmieder, B. 2006, A\&A, 459, 651

Suleiman, R. M., Crooker, N. U., Raymond, J. C., \& van Ballegooijen, A. 2005, in Coronal, Stellar Mass Ejections (Cambridge: Cambr. Univ. Press), IAU Symp., 226, 71

Thompson, W. T. 2009, Icarus., 200, 351

Thompson, W. T. 2011, JASTP, in press

Török, T., Kliem, B., \& Titov, V. S. 2004, A\&A, 413, L27

Wuelser, J.-P., Lemen, J. R., Tarbell, T. D., et al. 2004, Proc. SPIE, 5171, 111

Yurchyshyn, V., Abramenko, V., \& Tripathi, D. 2009, ApJ, 705, 426

Zhou, G. P., Wang, J. X., Zhang, J., et al. 2006, ApJ, 651, 1238 